\title{
Root Dimensions in Patients with Mild Hypodontia and a Control Group
}

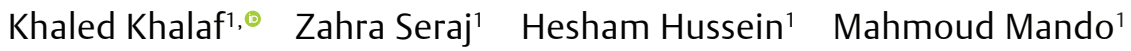 \\ ${ }^{1}$ Department of Preventive and Restorative Dentistry, University of \\ Sharjah, Sharjah, United Arab Emirates

\begin{abstract}
Address for correspondence Khaled Khalaf, PhD, BDS, DAS (Orth.), FDS (Orth) RCS (Eng), FFDRCSI (Orth.), M Orth RCS (Eng), M Orth RCS (Edin), MFDSRCS (Eng), MFDSRCS (Edin), MFDSRCPS (Glasg), FHEA (UK), FDTFEd, College of Dental Medicine (Building M28), University of Sharjah, PO Box 27272, Sharjah, United Arab Emirates (e-mail: kkhalaf@sharjah.ac.ae).
\end{abstract}

Eur J Dent 2019;13:574-580

\begin{abstract}
Keywords

- hypodontia

- mild

-root

- dimension

- control

Objectives The aim of this study was to compare root dimensions (length and mesiodistal widths) between subjects with mild hypodontia and an age- and sexmatched control group.

Materials and Methods Root dimension measurements of all permanent teeth excluding third molars were made on standardly taken orthopantomograms of 50 individuals ( 25 hypodontia and 25 controls) attending the University of Sharjah Dental Hospital. The length and two mesiodistal widths were measured for each fully formed root. The length of the root was measured digitally by drawing a line from the midpoint and bisecting the mesiodistal cemento-enamel junction (CEJ) of the tooth and extended to its apex. The mesiodistal widths of each root were measured at the cervical region and at half way of and perpendicular to the length of the root.

Statistical Analysis Two sample $t$-tests were used to compare root dimension measurements between the hypodontia and control groups.

Results There were no significant differences between genders with regard to root length or widths measurements, and therefore genders were combined for further analysis. Patients with hypodontia have significantly shorter root lengths than controls for the upper central incisors, upper canines, first premolars, and lower first molars $(p<0.05)$. Similarly, root width at the midpoint of the root was found to be less in hypodontia group than that in controls for the upper central incisors, lower first premolars, upper first molars, and all second premolars $(p<0.05)$. Similar pattern of differences was found with regard to the root width at the cervical region $(p<0.05)$. Conclusions Patients with hypodontia have shorter and narrower roots of the whole permanent dentition except the upper lateral incisors, lower incisors, lower canines, and all second molars when compared with controls. In effect, this may affect the orthodontic treatment planning and implant placement.
\end{abstract}

\section{Introduction}

Hypodontia, also known as "selective tooth agenesis" or "congenital tooth absence," is the condition in which one to six teeth excluding the third molars are congenitally missing. ${ }^{1,2}$ It is the most common developmental anomaly in humans with a reported range of $4.4 \%$ to $13.4 \%,{ }^{2,3}$ occurring more commonly among females. ${ }^{2,3}$

The exact etiology of hypodontia is unknown, but it is multifactorial in nature ${ }^{4-6}$ and can be affected by genetic and environmental factors. ${ }^{1,2,6,7}$ It may arise as part of a recognized genetic syndrome or more commonly as a solitary
DOI https://doi.org/

10.1055/s-0039-1700658

ISSN 1305-7456.
License terms

(ㅇ) (1) $\ominus \circledast$ 
non-syndromic anomaly. ${ }^{7}$ Nonsyndromic hypodontia most commonly affects the permanent dentition, with the mandibular second premolars having the highest incidence, followed by the maxillary lateral incisors. ${ }^{7}$

Hypodontia may be classified as either complete and partial; or according to the number of congenitally missing teeth, as mild ( 2 teeth or less congenitally missing), moderate (3 to 5 teeth congenitally missing), and severe ( 6 or more teeth congenitally missing), ${ }^{3,6,8}$ The latter is also known as oligodontia. The most severe form of tooth agenesis occurs in the absence of the entire primary or permanent dentition and is termed anodontia. However, anodontia rarely occurs without an accompanying genetic disorder such as ectodermal dysplasia. ${ }^{9,10}$

Patients with hypodontia may present with disturbances in facial growth patterns, several features of malocclusion including midline deviations and deep overbites which may result in a loss of function, decreased aesthetics, and oral health-related quality of life issues., ${ }^{411-15}$ Management of patients with hypodontia is challenging and requires an interdisciplinary approach, careful treatment planning, longterm maintenance, and family counselling. ${ }^{5}$ Treatment may include strategic extraction of primary teeth, up-righting and aligning teeth orthodontically, and placement of intra-bony implants. ${ }^{16}$ Other treatment options may include autotransplantation and protraction of third molars. ${ }^{16}$

An association between hypodontia and reduced tooth crown dimensions of the remaining dentition has been reported by some studies, ${ }^{8}$ and a positive correlation between the severity of hypodontia and the extent of reduction in tooth crown dimensions has further been detected. ${ }^{8} \mathrm{Howev}-$ er, to date, there have been no studies in the literature that have comprehensively investigated the effects of hypodontia on tooth root dimensions of the remaining dentition. Therefore, the aim of this study was to compare root dimension measurements between subjects with mild hypodontia and an age- and sex-matched control group. The information obtained from this study may aid in the understanding of the etiology of hypodontia and facilitate the diagnosis, treatment planning, and management of these complex cases.

\section{Materials and Methods}

\section{Participants}

Ethical approved was sought and granted from Research Ethics Committee at University of Sharjah to use patient records from the University Dental Hospital Sharjah (Reference number: REC-18-02-12-04-S).
A sample size calculation was performed for this casecontrol study and it was determined that 20 subjects would be required in each group to detect a clinically significant difference of $0.9 \mathrm{~mm}$ with $0.05 \alpha$ and $0.2 \beta$. The study sample ( - Fig. 1) consisted of 50 individuals: 25 hypodontia patients and 25 age- and sex-matched controls, with full complement of the permanent dentition. Each subgroup consisted of 14 males and 11 females. All hypodontia subjects were selected from the University Dental Hospital Sharjah database, Sharjah, UAE, and the controls were selected from the same database, ensuring each hypodontia subject was matched with a control in terms of age and gender. Subjects clinical notes and orthopantomograms (OPTs) were examined to ensure the details were correct and the inclusion criteria were met. The inclusion criteria for hypodontia subjects were as follows:

- No general medical conditions or syndromes

- Had no supernumerary teeth

- Had completely formed roots of the permanent dentition excluding third molars

- No previous orthodontic treatment

- Had 1-2 teeth congenitally absent excluding the third molars.

The inclusion criteria for control subjects were the same as for hypodontia subjects, except control subjects should have had no congenital absence of the permanent teeth.

The age range for the whole sample was 12 to 20 years, and the mean age range for both groups was 14.92 years with a standard deviation (SD) of 2.91 years.

\section{Measurements Recorded}

Root dimension measurements ( - Fig. 2) of all permanent teeth excluding third molars were made on standardly taken OPTs of all subjects on MiPACS Dental Enterprise Solution ${ }^{\mathrm{TM}}$. Measurements made were as follows:

1. The mesiodistal width $(\mathrm{A})$ at the cemento-enamel junction (CEJ): This was measured digitally with the aid of the "ruler" tool up to the second decimal digit.

2. The length $(B)$ was defined as the maximum length between the root apex and the midpoint of measurement $\mathrm{A}$.

3. The second mesiodistal width (C) was measured by drawing a line at halfway and perpendicular to the length of the root (B).

All measurements were performed by one trained operator twice and the mean value of the two measurements was recorded. The measurements error was assessed by

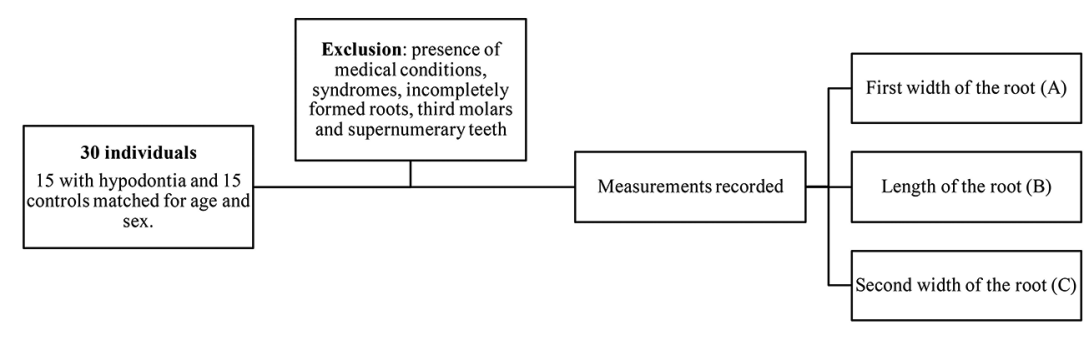

Fig. 1 Study sample selection and measurements recorded. 

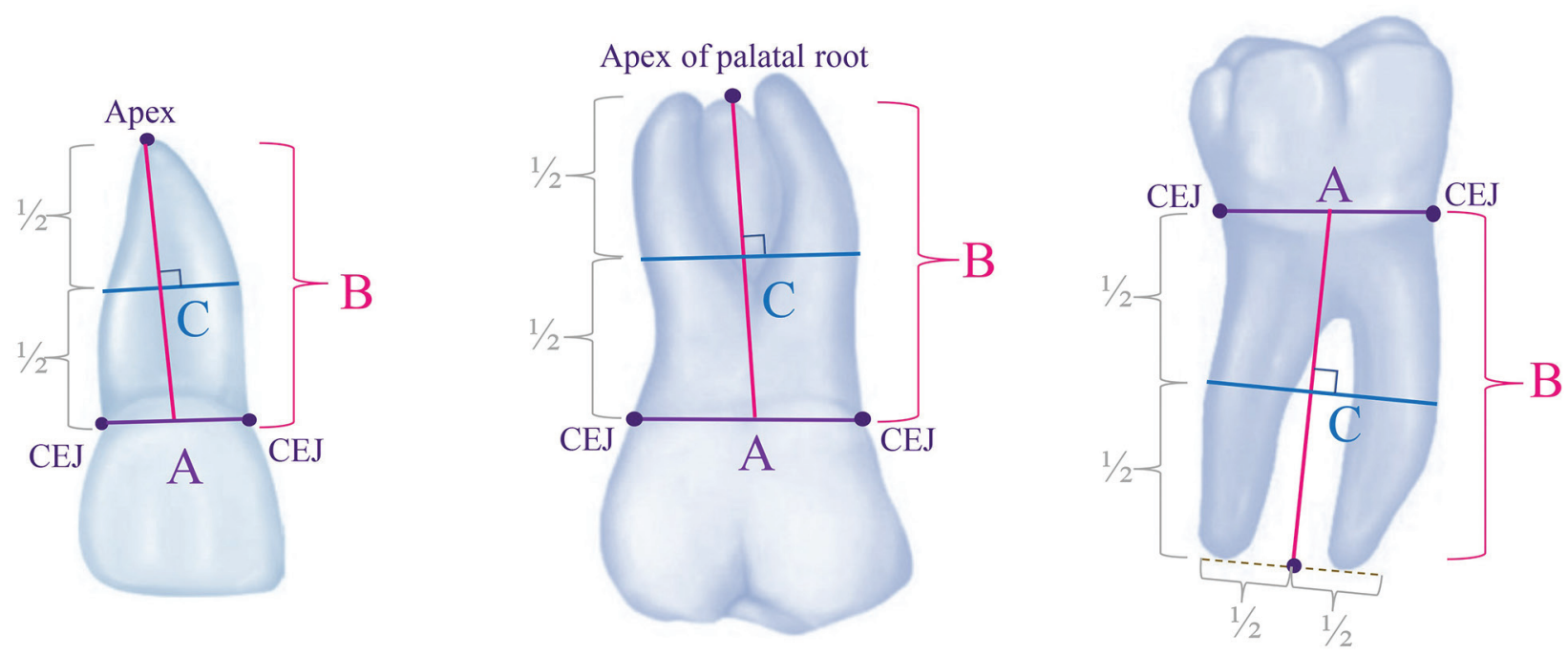

Fig. 2 Root dimension measurements for a (i) single rooted tooth, (ii) upper multi-rooted tooth and (iii) lower multi rooted tooth. CEJ, cemento-enamel junction.

carrying out the above measurements twice 8 weeks apart on six tooth types ( 3 from the maxillary arch and 3 from the mandibular arch) on 20 randomly selected OPTs, of which 10 were hypodontia patients and 10 were control subjects. No statistically significant difference was detected between the two measurements using a paired sample $t$-test $(p>0.05)$. The method error was assessed using Dahlberg formula. The range of the measurements error between the double measurements was found to be 0.09 to $0.25 \mathrm{~mm}$, which was considered very small when compared with the average values of the measurements in the study.

\section{Statistical Analysis}

Statistical analyses were performed using SPSS Ver. 24.0 for Mac OS X. The data were found to be normally distributed ( $p<0.05$; Kolmogorov-Smirnov test); therefore, parametric testing was applied. Two-sample $t$-tests were applied to compare hypodontia and control groups. Differences were considered statistically significant at $p<0.05$.

\section{Results}

-Table 1 and -Fig. 3 show comparison of root dimension measurements $\mathrm{A}, \mathrm{B}$, and $\mathrm{C}$ of the permanent maxillary teeth between the hypodontia and control groups. - Table 2 and

-Fig. 4 show comparison of root dimension measurements $\mathrm{A}, \mathrm{B}$ and $\mathrm{C}$ of the permanent mandibular teeth between the hypodontia and control groups. Hypodontia patients had significantly smaller mesiodistal root dimension A of the maxillary first and second premolars, maxillary first molars, and mandibular second premolars $(p<0.05)$. Hypodontia subjects also demonstrated significantly shorter root dimensions B of the maxillary central incisors, maxillary canines, maxillary first premolars, and mandibular first molars $(p<0.05)$. Additionally, they had reduced mesiodistal root dimension $C$ of the maxillary central incisors, maxillary second premolars, maxillary first molars, and mandibular first and second premolars $(p<0.05)$.

\section{Discussion}

To date, our study is the first to report root dimension measurements in mild hypodontia patients, and therefore it is not possible to compare our findings regarding root dimension measurements in hypodontia patients with others. The most commonly congenitally missing teeth found in our study sample were the mandibular second premolars and the maxillary lateral incisors, followed by the mandibular lower incisors and the maxillary second premolars (-Fig. 5), which agrees with most previous investigations of the distribution of the congenitally missing teeth of the permanent dentition. ${ }^{3-5}$ As can be seen from - Tables 1 and 2 and -Figs. 3 and $\mathbf{4}$, the teeth which were most affected with a reduction in root length in the hypodontia group, as compared with the control group, were mainly in the maxillary arch. More specifically, they were located more mesial of each morphological class in the maxillary arch, that is, the upper central incisors, upper canines, and upper first premolars. This finding does not agree with Butler's morphological field theory, ${ }^{17}$ as applied to the human dentition, ${ }^{18}$ which suggests that the tooth which is positioned more mesially of each morphological class is more under genetic control and less variable than the remaining teeth of the same class. However, more recent studies investigating the etiology of anomalies of tooth number and size have suggested a more comprehensive and clinically relevant explanation of the association of anomalies of tooth number and size, where genetic, epigenetic, and environmental factors were all thought to be involved with varying degrees of influences depending on the individuals. ${ }^{6,19,20}$ In our study, the teeth which were found to be affected in the hypodontia group have longer roots than the teeth which were not affected. Therefore, it may well be that there were more chances for the epigenetic and environmental factors to play their roles and hinder their development in the hypodontia group as compared with the unaffected teeth of shorter roots. 
Table 1 Comparison of root dimension measurements of the permanent maxillary teeth in hypodontia patients and controls

\begin{tabular}{|c|c|c|c|c|}
\hline Tooth & Measurement & Hypodontia (mean \pm SD) & Controls (mean \pm SD) & $p$-Value \\
\hline \multirow[t]{3}{*}{ U1 } & A & $6.94 \pm 1.22$ & $7.51 \pm 0.79$ & 0.055 \\
\hline & B & $17.05 \pm 1.98$ & $18.65 \pm 1.73$ & 0.005 \\
\hline & C & $5.53 \pm 0.98$ & $6.26 \pm 0.71$ & 0.005 \\
\hline \multirow[t]{3}{*}{ U2 } & A & $6.04 \pm 1.04$ & $6.08 \pm 0.57$ & 0.902 \\
\hline & B & $17.07 \pm 1.90$ & $18.03 \pm 1.80$ & 0.094 \\
\hline & C & $4.82 \pm 0.84$ & $5.05 \pm 0.44$ & 0.296 \\
\hline \multirow[t]{3}{*}{ U3 } & A & $7.50 \pm 0.90$ & $8.01 \pm 1.65$ & 0.343 \\
\hline & B & $18.43 \pm 3.31$ & $21.73 \pm 2.78$ & 0.007 \\
\hline & $\mathrm{C}$ & $5.88 \pm 1.32$ & $6.44 \pm 1.06$ & 0.211 \\
\hline \multirow[t]{3}{*}{ U4 } & A & $7.10 \pm 1.04$ & $8.49 \pm 0.68$ & 0.038 \\
\hline & B & $15.49 \pm 1.55$ & $17.47 \pm 2.49$ & 0.015 \\
\hline & $C$ & $5.75 \pm 1.02$ & $7.12 \pm 0.81$ & 0.039 \\
\hline \multirow[t]{3}{*}{ U5 } & A & $7.29 \pm 0.61$ & $8.12 \pm 0.78$ & 0.006 \\
\hline & $\mathrm{B}$ & $15.57 \pm 2.00$ & $17.02 \pm 2.73$ & 0.137 \\
\hline & $\mathrm{C}$ & $5.64 \pm 0.81$ & $6.35 \pm 0.93$ & 0.048 \\
\hline \multirow[t]{3}{*}{ U6 } & A & $10.19 \pm 1.60$ & $11.03 \pm 0.82$ & 0.026 \\
\hline & B & $15.98 \pm 2.34$ & $17.21 \pm 2.22$ & 0.076 \\
\hline & $\mathrm{C}$ & $8.15 \pm 1.52$ & $9.50 \pm 1.21$ & 0.002 \\
\hline \multirow[t]{3}{*}{ U7 } & A & $9.98 \pm 0.84$ & $10.41 \pm 0.78$ & 0.262 \\
\hline & $\mathrm{B}$ & $15.16 \pm 1.99$ & $15.07 \pm 3.74$ & 0.944 \\
\hline & $C$ & $8.00 \pm 0.84$ & $8.32 \pm 0.70$ & 0.383 \\
\hline
\end{tabular}

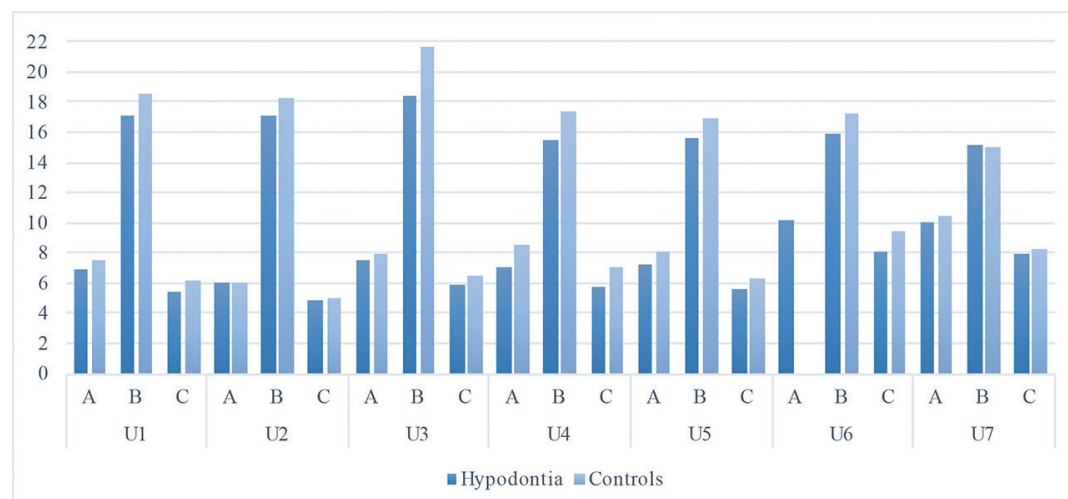

Fig. 3 Root dimension measurements of the maxillary teeth in hypodontia patients and controls.

With regard to widths measurements, a similar pattern to the differences in root measurements mentioned above was also observed here, but with some degrees of variation; thus, providing more support to the multifactorial etiology of anomalies of tooth number and size..$^{6,19,20}$ Furthermore, it was found that the number of teeth which had a reduction in root width at half way of the root was more than those which had a reduction in root width at the cervical region. This is expected as the cervical region forms and calcifies well before the middle area of the root and therefore it was more likely to have less influential epigenetic and environmental variables during dental development.

It is of note that the maxillary lateral incisors, mandibular lower incisors, and mandibular canines were neither affected in root length dimensions nor in root widths measurements. This may be attributed to the simpler morphology and relatively smaller sizes of their roots than those of the remaining teeth.

Patients with hypodontia often present with various features of malocclusion, and require multidisciplinary management involving orthodontic treatment to close, redistribute, and/or open spaces, followed by prosthetic replacement of the congenitally missing teeth and/or restorative reshaping of the peg-shaped/microdontic teeth in the anterior regions. ${ }^{16,21-25}$ As hypodontia patients may have shorter and narrower roots than the average population, it is important to carefully plan the anchorage in hypodontia patients and reinforce it with several means, so that the desired tooth 
Table 2 Comparison of root dimension measurements of the permanent mandibular teeth in hypodontia patients and controls

\begin{tabular}{|c|c|c|c|c|}
\hline Tooth & Measurement & Hypodontia (mean \pm SD) & Controls (mean \pm SD) & $p$-Value \\
\hline \multirow[t]{3}{*}{ L1 } & $A$ & $4.62 \pm 0.69$ & $4.66 \pm 0.41$ & 0.81 \\
\hline & $\mathrm{B}$ & $14.51 \pm 1.77$ & $15.29 \pm 2.04$ & 0.162 \\
\hline & $\mathrm{C}$ & $3.99 \pm 1.23$ & $4.00 \pm 1.21$ & 0.98 \\
\hline \multirow[t]{3}{*}{ L2 } & A & $4.79 \pm 0.72$ & $5.10 \pm 0.60$ & 0.116 \\
\hline & $\mathrm{B}$ & $15.05 \pm 1.54$ & $15.78 \pm 1.87$ & 0.156 \\
\hline & C & $3.94 \pm 0.64$ & $4.26 \pm 0.54$ & 0.062 \\
\hline \multirow[t]{3}{*}{ L3 } & $A$ & $7.00 \pm 1.12$ & $7.13 \pm 0.71$ & 0.691 \\
\hline & $\mathrm{B}$ & $18.39 \pm 1.83$ & $19.64 \pm 3.37$ & 0.223 \\
\hline & C & $6.26 \pm 1.14$ & $6.68 \pm 0.91$ & 0.263 \\
\hline \multirow[t]{3}{*}{ L4 } & A & $6.63 \pm 0.90$ & $7.02 \pm 0.58$ & 0.148 \\
\hline & $\mathrm{B}$ & $16.89 \pm 1.31$ & $17.53 \pm 1.92$ & 0.274 \\
\hline & C & $5.12 \pm 0.70$ & $5.63 \pm 0.47$ & 0.021 \\
\hline \multirow[t]{3}{*}{ L5 } & A & $6.97 \pm 1.02$ & $8.03 \pm 0.87$ & 0.012 \\
\hline & B & $17.00 \pm 3.04$ & $18.45 \pm 3.33$ & 0.272 \\
\hline & C & $5.48 \pm 0.80$ & $6.34 \pm 0.88$ & 0.023 \\
\hline \multirow[t]{3}{*}{ L6 } & A & $11.31 \pm 1.64$ & $11.75 \pm 0.89$ & 0.252 \\
\hline & B & $16.54 \pm 2.34$ & $17.97 \pm 1.98$ & 0.027 \\
\hline & $C$ & $11.07 \pm 1.58$ & $11.68 \pm 0.88$ & 0.105 \\
\hline \multirow[t]{3}{*}{ L7 } & A & $11.76 \pm 0.57$ & $12.38 \pm 0.61$ & 0.031 \\
\hline & B & $14.64 \pm 3.07$ & $15.79 \pm 3.35$ & 0.438 \\
\hline & C & $10.80 \pm 1.14$ & $11.03 \pm 3.07$ & 0.726 \\
\hline
\end{tabular}

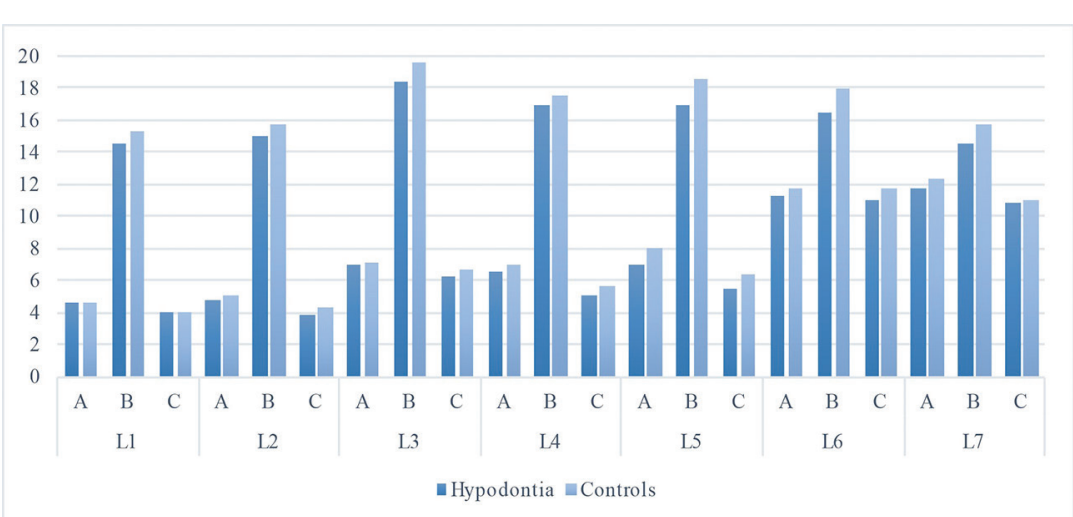

Fig. 4 Root dimension measurements of the mandibular teeth in hypodontia patients and controls.

movements can be achieved with no untoward effects. ${ }^{22}$ Furthermore, it has been reported that short and narrow roots were at greater risk of root resorption during orthodontic treatment, especially the maxillary incisors. ${ }^{26-29}$ Therefore, in hypodontia patients, an optimal force system should be applied during orthodontic treatment, with close monitoring to avoid significant root resorption, especially, of the maxillary central incisors.

Moreover, it should be borne in mind that shorter and narrower size implants may suffice in hypodontia patients during the planning phase to prosthetically replace the congenitally missing teeth following the orthodontic treatment. ${ }^{30,31}$ This is especially so, as patients with hypodontia were also reported to have smaller clinical crowns of the remaining dentition when compared with controls, ${ }^{8}$ and inadequate bone to house the standard-sized implants. ${ }^{23,32}$

The findings of our study should be interpreted with some caution due to some limitations as is often the case with the majority of studies. In our study, we assessed root dimension measurements on orthopantomograms, which are $2 \mathrm{D}$ projections of the teeth with a reported average magnification error of 15 to $25 \% .^{33-35}$ However, as our study was a comparison case-control study with all OPTs taken by one trained operator using a standard method, the comparison should still remain valid. Furthermore, hypodontia patients in our study were all of mild severity, having only one or two teeth congenitally missing; thus, the findings can only apply for mild hypodontia patients. 


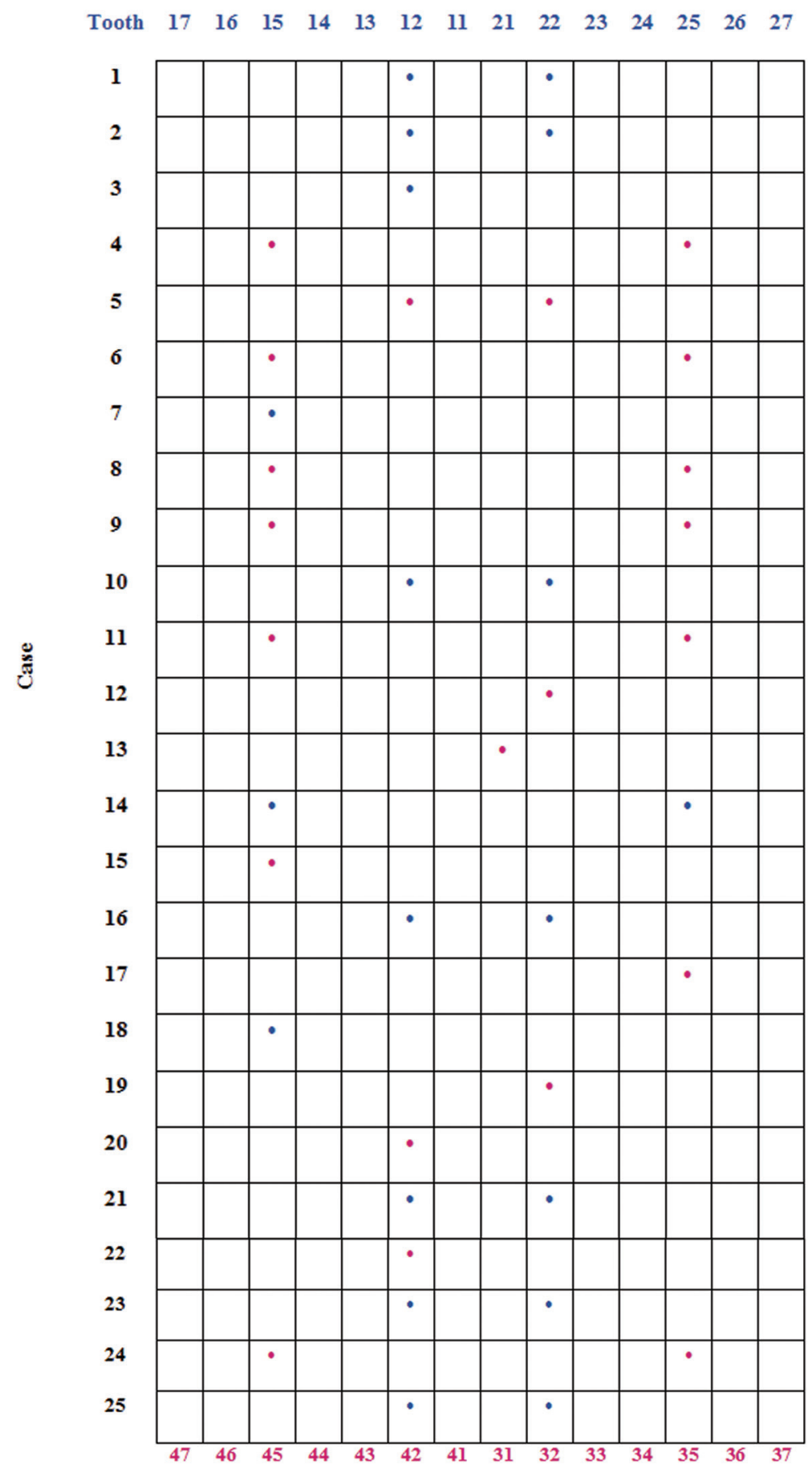

Fig. 5 Pattern of missing teeth in hypodontia cases.

Further research should investigate the impact of the severity of hypodontia on root dimension measurements using three-dimensional imaging, as it may well be that the more severe the hypodontia the more reduction in root dimension measurements. A similar finding has already been reported with regard to size of the clinical crowns of the permanent dentition. ${ }^{8}$

\section{Conclusions}

Patients with mild hypodontia may have shorter and narrower roots of the whole permanent dentition, except the upper lateral incisors, lower incisors, lower canines, and all second molars, when compared with controls. In effect, this may affect the orthodontic treatment planning and implant placement. 


\section{Funding}

None.

\section{Conflict of Interest}

None declared.

\section{References}

1 Uzuner D, Celik MM, Toy E, Turkdonmez CO. Assessment of hypodontia in the Turkish patients referring to the orthodontic clinic: A retrospective study. Eur J Dent 2013;7(Suppl 1):S9-S14

2 Cobourne MT, Sharpe PT. Diseases of the tooth: the genetic and molecular basis of inherited anomalies affecting the dentition. Wiley Interdiscip Rev Dev Biol 2013;2(2):183-212

3 Khalaf K, Miskelly J, Voge E, Macfarlane TV. Prevalence of hypodontia and associated factors: a systematic review and meta-analysis. J Orthod 2014;41(4):299-316

4 Rakhshan V. Congenitally missing teeth (hypodontia): A review of the literature concerning the etiology, prevalence, risk factors, patterns and treatment. Dent Res J (Isfahan) 2015;12(1):1-13

5 Mattheeuws N, Dermaut L, Martens G. Has hypodontia increased in Caucasians during the 20th century? A meta-analysis. Eur J Orthod 2004;26(1):99-103

6 Brook AH. Multilevel complex interactions between genetic, epigenetic and environmental factors in the aetiology of anomalies of dental development. Arch Oral Biol 2009;54 (Suppl 1):S3-S17

7 Galluccio G, Castellano M, La Monaca C. Genetic basis of non-syndromic anomalies of human tooth number. Arch Oral Biol 2012;57(7):918-930

8 Khalaf K. Tooth size in patients with mild, moderate and severe hypodontia and a control group. Open Dent J 2016;10:382-389

9 Hsieh YL, Razzoog M, Garcia Hammaker S. Oral care program for successful long-term full mouth habilitation of patients with hypohidrotic ectodermal dysplasia. Case Rep Dent 2018;2018:4736495

10 Bailleul-Forestier I, Molla M, Verloes A, Berdal A. The genetic basis of inherited anomalies of the teeth. Part 1: clinical and molecular aspects of non-syndromic dental disorders. Eur J Med Genet 2008;51(4):273-291

11 Laing E, Cunningham SJ, Jones S, Moles D, Gill D. Psychosocial impact of hypodontia in children. Am J Orthod Dentofacial Orthop 2010;137(1):35-41

12 Locker D, Jokovic A, Prakash P, Tompson B. Oral health-related quality of life of children with oligodontia. Int J Paediatr Dent 2010;20(1):8-14

13 Kotecha S, Turner PJ, Dietrich T, Dhopatkar A. The impact of tooth agenesis on oral health-related quality of life in children. J Orthod 2013;40(2):122-129

14 Karadas M, Celikoglu M, Akdag MS. Evaluation of tooth number anomalies in a subpopulation of the North-East of Turkey. Eur J Dent 2014;8(3):337-341

15 Wong AT, McMillan AS, McGrath C. Oral health-related quality of life and severe hypodontia. J Oral Rehabil 2006;33(12):869-873

16 Gill DS, Barker CS. The multidisciplinary management of hypodontia: a team approach. Br Dent J 2015;218(3):143-149

17 Cakan DG, Ulkur F, Taner T. The genetic basis of dental anomalies and its relation to orthodontics. Eur J Dent 2013;7(1, Suppl 1):S143-S147
18 Dahlberg AA. The changing dentition of man. J Am Dent Assoc 1945;32:676-690

19 Townsend GC, Brook AH. Genetic, epigenetic and environmental influences on dental development. Ortho Tribune 2008;3:3-6

20 Townsend G, Harris EF, Lesot H, Clauss F, Brook A. Morphogenetic fields within the human dentition: a new, clinically relevant synthesis of an old concept. Arch Oral Biol 2009;54(Suppl 1):S34-S44

21 Nunn JH, Carter NE, Gillgrass TJ, et al. The interdisciplinary management of hypodontia: background and role of paediatric dentistry. Br Dent J 2003;194(5):245-251

22 Carter NE, Gillgrass TJ, Hobson RS, et al. The interdisciplinary management of hypodontia: orthodontics. Br Dent J 2003;194(7):361-366

23 Jepson NJ, Nohl FS, Carter NE, et al. The interdisciplinary management of hypodontia: restorative dentistry. $\mathrm{Br}$ Dent J 2003;194(6):299-304

24 Meechan JG, Carter NE, Gillgrass TJ, et al. Interdisciplinary management of hypodontia: oral surgery. Br Dent J 2003;194(8):423-427

25 Hobson RS, Carter NE, Gillgrass TJ, et al. The interdisciplinary management of hypodontia: the relationship between an interdisciplinary team and the general dental practitioner. $\mathrm{Br}$ Dent J 2003;194(9):479-482

26 Picanço GV, de Freitas KM, Cançado RH, Valarelli FP, Picanço PR, Feijão CP. Predisposing factors to severe external root resorption associated to orthodontic treatment. Dental Press J Orthod 2013;18(1):110-120

27 Elhaddaoui R, Benyahia H, Azeroual MF, Zaoui F, Razine $\mathrm{R}$, Bahije L. Resorption of maxillary incisors after orthodontic treatment-clinical study of risk factors. Int Orthod 2016;14(1):48-64

28 Nigul K, Jagomagi T. Factors related to apical root resorption of maxillary incisors in orthodontic patients. Stomatologija 2006;8(3):76-79

29 Lopatiene K, Dumbravaite A. Risk factors of root resorption after orthodontic treatment. Stomatologija 2008;10(3):89-95

30 King P, Maiorana C, Luthardt RG, et al. Clinical and radiographic evaluation of a small-diameter dental implant used for the restoration of patients with permanent tooth agenesis (hypodontia) in the maxillary lateral incisor and mandibular incisor regions: a 36-month follow-up. Int J Prosthodont 2016;29(2):147-153

31 Bahrami M, Saleh Saber F, Hendi A. Comprehensive treatments for congenitally missing teeth and generalized diastema. Case Rep Dent 2017;2017:3254873

32 Forgie AH, Thind BS, Larmour CJ, Mossey PA, Stirrups DR. Management of hypodontia: restorative considerations. Part III. Quintessence Int 2005;36(6):437-445

33 Sanderink GC, Visser WN, Kramers EW. The origin of a case of severe image distortion in rotational panoramic radiography. Dentomaxillofac Radiol 1991;20(3):169-171

34 Choi YG, Kim YK, Eckert SE, Shim CH. Cross-sectional study of the factors that influence radiographic magnification of implant diameter and length. Int J Oral Maxillofac Implants 2004;19(4):594-596

35 Samawi SS, Burke PH. Angular distortion in the orthopantomogram. Br J Orthod 1984;11(2):100-107 